\title{
Epidemiology and pathology of avian malaria in penguins undergoing rehabilitation in Brazil
}

\author{
Ralph Eric Thijl Vanstreels ${ }^{1 *}$, Rodolfo Pinho da Silva-Filho ${ }^{2}$, Cristiane Kiyomi Miyaji Kolesnikovas ${ }^{3}$, \\ Renata Cristina Campos Bhering ${ }^{4}$, Valeria Ruoppolo ${ }^{1,5}$, Sabrina Epiphanio ${ }^{6}$, Marcos Amaku \\ Francisco Carlos Ferreira Junior ${ }^{8}$, Érika Martins Braga ${ }^{8}$ and José Luiz Catão-Dias ${ }^{1}$
}

\begin{abstract}
Seabird rehabilitation is a valuable strategy to mitigate the impacts of oil pollution and other anthropogenic factors, and can significantly contribute to the conservation of penguins. However, infectious diseases such as avian malaria (Plasmodium spp.) can hamper the success of rehabilitation efforts. We combined morphological and molecular diagnostic methods to investigate the epidemiology and pathology of Plasmodium in Magellanic penguins (Spheniscus magellanicus) at rehabilitation centers along $2500 \mathrm{~km}$ of the coastline of Brazil. True prevalence of malarial parasites was estimated between $6.6 \%$ and $13.5 \%$. We identified five species, three of which had not been described infecting penguins (P. cathemerium, P. nucleophilum, P. unalis); an additional five distinct Plasmodium lineages were also distinguished, and albeit unidentified these clearly correspond to species that also have not yet been reported in penguins. Our results indicate that the diversity of plasmodia that may infect these birds is greater than previously recognised. Considering the well-defined seasonality observed in this study, it is clear that rehabilitation centers could benefit by narrowing their preventative efforts on penguins maintained or admitted during the Austral spring-summer, particularly by preventing mosquitoes from coming into contact with penguins.
\end{abstract}

\section{Introduction}

Avian malaria is a disease caused by mosquito-transmitted protozoans of the genus Plasmodium, and more than 60 species are known to infect birds [1]. Plasmodium infections tend to be asymptomatic or pose only minor impact on fitness and survival of most species of birds, however a few avian groups are considered highly susceptible and may develop severe disease when exposed to these parasites [2]. Penguins (Sphenisciformes) are one such group, and avian malaria is an important infectious diseases for these birds, especially in a captive environment $[3,4]$. Nearly all cases of avian malaria in penguins have been attributed to $P$. relictum and/or P. elongatum $[3,5,6]$, with only isolated reports of infection by $P$. juxtanucleare [7] and P. tejerai [8].

Magellanic penguins (Spheniscus magellanicus) breed along the coast of Argentina, Chile and the Malvinas-

\footnotetext{
*Correspondence: ralph_vanstreels@yahoo.com.br

'Departamento de Patologia, Faculdade de Medicina Veterinária e Zootecnia, Laboratório de Patologia Comparada de Animais Selvagens, Universidade de São Paulo, Avenida Orlando Marques de Paiva 87, São Paulo, SP, 05088-000, Brazil

Full list of author information is available at the end of the article
}

Falkland Islands [9], and are susceptible to the disease. There are reports of Plasmodium sp infections in Magellanic penguins (MPs) at zoos in the United States [10], South Korea [11] and Brazil [12], and at rehabilitation centers in Chile [13] and Brazil [8,14]. In each of these reports, avian malaria has led to rapid outbreaks with high morbidity and mortality of MPs. In contrast, none of the studies examining blood or tissue samples of wild MPs have found evidence of Plasmodium sp or other blood parasites, nor have studies on sympatric populations of other penguin species $[5,6]$.

The fact that there are reports of Plasmodium sp in MPs in captivity and undergoing rehabilitation but not in the wild suggests the MPs are Plasmodium-free when admitted to rehabilitation centers, and acquire the infection during their time in these facilities. We examine this hypothesis by conducting a broad investigation for Plasmodium spp. in MPs at rehabilitation centers along the coast of Brazil, determining spatial and temporal distribution of malarial infections, mortality and lineages. 


\section{Materials and methods}

\section{Study locations and data collection}

We studied MPs received for rehabilitation at five organizations along the coast of Brazil (Figure 1): CRAMFURG (Rio Grande, Rio Grande do Sul - 3201'34"S 52 06’21”W), CETAS Florianópolis (Florianópolis, Santa

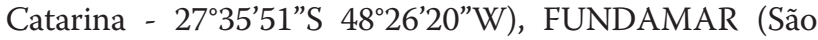
Sebastião, São Paulo - 2349'21"S 45²4'53”W), CETAS Unimonte (São Vicente, São Paulo - 2356'50"S $46^{\circ}$ 23’39”W), and IPRAM (Cariacica, Espírito Santo - 20 $\left.19^{\prime} 54^{\prime \prime S} 40^{\circ} 21^{\prime} 38^{\prime \prime W}\right)$. Each organization receives penguins rescued along the coastline of their state and neighboring states; additionally, CRAM-FURG also receives then releases penguins that were rehabilitated at other states (Bahia, Espírito Santo, Rio de Janeiro). Considering this dynamic, each penguin was assigned a "location" (Bahia, Espírito Santo, Rio de Janeiro, São Paulo, Santa Catarina, Rio Grande do Sul) based on the facility in which they were subjected to rehabilitation (not rescue nor release location).

Samples were collected in different time periods at each location (Table 1). Samples were collected systematically from January 2009 to December 2012 at Rio Grande do Sul and from March 2009 to February 2013 at Santa Catarina; in these periods, MPs were evenly sampled without bias towards clinically ill or healthy individuals and over an extended time period ("systematic sampling"). Other samples were collected from other facilities over a short period and/or from penguins showing clinical or necropy findings suggestive of infectious disease ("opportunistic sampling").

Samples and biological information collected within 0-3 days from intake to the facility were considered "intake", whereas those collected 0-7 days before death or release were considered "exit". Each individual was categorised in relation to their "age group" on intake (juvenile, adult), "oiling" on intake (oiled, not oiled), "survival" during rehabilitation (survivor, deceased) and "diagnosis" (positive, negative). Rehabilitation records were used to determine "warm season period", i.e. the number of days spent in the facility (from intake to death or release) that occurred within the period 01 October to 31 March.

\section{Study design}

Screening for Plasmodium combined one or more of the following diagnostic methods: thin blood smears (TBS), nested polymerase chain reaction (PCR) and histopathology (HP).

Plasmodium screening was divided in two stages. The following criteria were adopted in the first stage: (a) all TBS collected at all study sites were examined; (b) for "survivor" penguins, the "exit" blood sample was tested with PCR; (c)

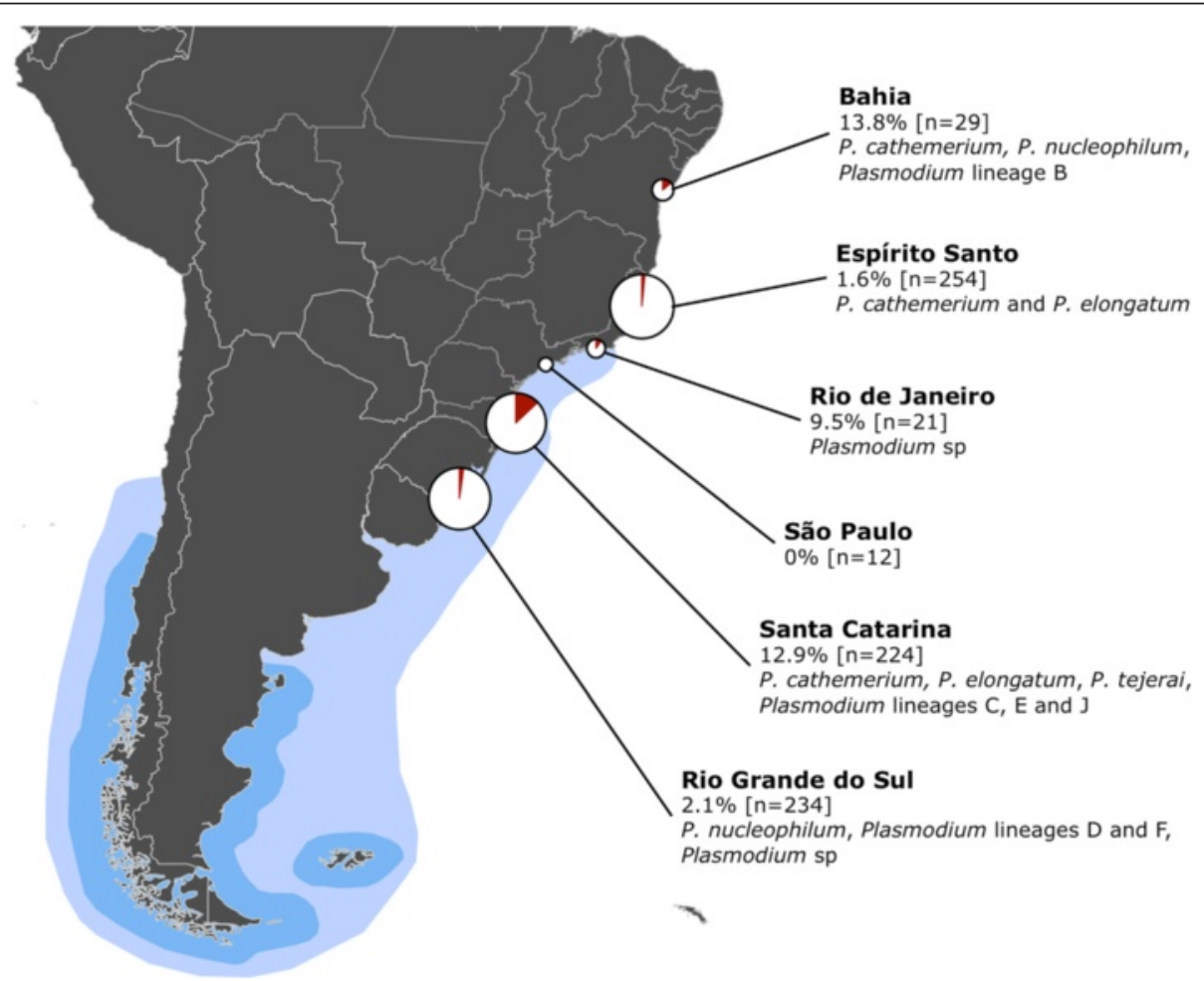

Figure 1 Geographic distribution of the sampling effort, detection and lineages of Plasmodium spp. Pie charts represent sampling effort (size) and percentage of positive results (red fraction). Blue areas represent the wintering (light blue) and breeding (darker blue) distribution of Magellanic penguins [9]. 
Table 1 Sample sizes examined using different diagnostic tests to screen for Plasmodium sp infections

\begin{tabular}{|c|c|c|c|c|c|c|}
\hline Latitude & Study site (time period) & Blood smears + PCR & PCR & Blood smears & Histopathology & Total \\
\hline $13^{\circ} 00^{\prime} \mathrm{s}$ & Bahia $(1999-2008)^{\ddagger}$ & $7(1)$ & - & $7(1)$ & - & $8(2)$ \\
\hline $13^{\circ} 00^{\prime} \mathrm{S}$ & Bahia (Jun2009-Dec2012) ${ }^{\ddagger}$ & $21(2)$ & - & - & - & $21(2)$ \\
\hline $20^{\circ} 20^{\prime} \mathrm{S}$ & Espírito Santo $(1999-2008)^{\ddagger}$ & 2 & - & $17(1)$ & - & $19(1)$ \\
\hline $20^{\circ} 20^{\prime} \mathrm{S}$ & Espírito Santo (Sep2012) & 86 & - & 111 & - & 197 \\
\hline $20^{\circ} 20^{\prime} \mathrm{S}$ & Espírito Santo (Sep2012-Feb2013) & $18(2)$ & $20(1)$ & - & - & $38(3)$ \\
\hline $22^{\circ} 50^{\prime} \mathrm{S}$ & Rio de Janeiro $(1999-2008)^{\ddagger}$ & 6 & $1(1)$ & 5 & $1(1)$ & $13(2)$ \\
\hline $22^{\circ} 50^{\prime} \mathrm{S}$ & Rio de Janeiro (Jan2009-Dec2012) & 2 & - & 6 & - & 8 \\
\hline $23^{\circ} 58^{\prime} \mathrm{S}$ & São Paulo (Aug2010-Sep2010) & 1 & 11 & - & - & 12 \\
\hline $27^{\circ} 36^{\prime} S$ & Santa Catarina (Mar2009-Feb2013) ${ }^{\dagger}$ & $106(19)$ & $81(8)$ & $37(2)$ & - & $224(29)$ \\
\hline $32^{\circ} 02^{\prime} \mathrm{S}$ & Rio Grande do Sul (1999-2008) & - & - & 11 & $2(2)$ & $13(2)$ \\
\hline $32^{\circ} 02^{\prime} \mathrm{S}$ & Rio Grande do Sul (Jan2009-Dec2012) ${ }^{\dagger}$ & $192(3)$ & 8 & 21 & - & $221(3)$ \\
\hline \multirow[t]{3}{*}{ Total } & Systematic sample collection & $298(22)$ & $89(8)$ & $58(2)$ & - & $445(32)$ \\
\hline & Opportunistic sample collection & $143(5)$ & $32(2)$ & $151(2)$ & $3(3)$ & $329(12)$ \\
\hline & Grand total & 441 (27) & $121(10)$ & 209 (4) & $3(3)$ & 774 (44) \\
\hline
\end{tabular}

Values within parenthesis indicate the number of positive samples. " $\dagger$ " indicates that sample collection was systematic, i.e. was not conducted in a manner that could favor sick or healthy individuals. " $\neq$ " indicates samples collected upon admission to CRAM-FURG from penguins that had been rehabilitated at other facilities.

for "deceased" penguins from which frozen tissue samples had been collected at necropsy, these tissues were tested with PCR; (d) for "deceased" penguins from which frozen tissue sampled were not collected at necropsy, the last blood sample collected before death was tested with PCR; (e) for "deceased" penguins from which neither blood nor frozen tissue samples were available, tissue samples in formalin were examined by HP. At this stage, blood smear examination was blind to PCR results and vice-versa.

The second stage of Plasmodium screening used the criteria: (a) if a penguin obtained a positive or inconclusive result for one or more samples in the first step, all samples available from that individual were tested with PCR; (b) if a penguin obtained a positive result, all samples from other individuals that had been at the same facility at the same date or three weeks before or following to the date of collection of the positive sample were tested with PCR. Because distinct sets of samples were available for each individual, different combinations of diagnostic tests were used to detect Plasmodium among study sites (Table 1).

Finally, individuals with positive results in the previous steps were further tested: (a) positive samples were subjected to sequencing of the $c y t-b$ gene; (b) histopathology of all available tissue samples were evaluated to determine microscopic lesions and determine the occurrence of exoerythrocytic meronts; (c) all available blood smears were used to characterize parasite morphology.

\section{Sample collection, hematology and pathology}

MPs at the study facilities are subjected to standardized rehabilitation protocols under the supervision of veterinarians [15]. At all facilities involved in the study, external and internal enclosures in which rehabilitation is conducted were not entirely protected against mosquitoes and were within 500 metres from bodies of freshwater and/or fragments of Atlantic forest. Penguins were physically restrained and blood samples were collected from the jugular or metatarsal veins. Body mass was determined with a scale with \pm 5 g precision; when these data were collected upon intake, it was referred to as "intake mass". Thin blood smears and heparin capillaries were prepared immediately after blood collection; the remaining blood was stored in tubes with heparin or without anticoagulants, then frozen. In some cases, hematocrit was determined through centrifugation in heparin capillaries at $16000 \mathrm{~g}$ for $5 \mathrm{~min}$; total plasma protein was determined with a clinical refractometer.

Blood smears were dried at room temperature, fixed with absolute methanol, stained with Giemsa or WrightRosenfeld stain, and examined under $1000 \times$ magnification (field of view area $=0.126 \mathrm{~mm}^{2}$ ). A minimum 150 fields ( 30000 erythrocytes) were examined during the first stage of screening and an additional 250 fields ( $\sim 50000$ erythrocytes) were examined during the second stage. Blood parasites were morphologically characterized [16] and quantified with the assistance of digital image analysis to count 10000 erythrocytes [17]; parasite forms were differentiated into four categories (trophozoite, meront, microgametocyte and macrogametocyte).

Whenever possible, penguins that died during rehabilitation were refrigerated and examined within 12 to $24 \mathrm{~h}$ after death; when this was not possible, carcasses were frozen for later examination. Gross lesions were photographed and noted, and samples of organs and tissues 
were fixed in 10\% buffered formalin. Formalin-fixed tissues were embedded in paraffin and sections of 3 or $5 \mu \mathrm{m}$ were obtained, stained with hematoxylin-eosin and examined under light microscopy.

\section{Molecular biology and phylogenetic analysis}

Frozen samples of blood and tissues (lung, spleen or liver) were used for molecular analyses. DNA extraction was conducted using the DNEasy Blood and Tissue Kit (\#69506, Qiagen) and was verified and quantified through UV spectrophotometry (Nanodrop 2000, Thermo Fisher Scientific). We used a nested polymerase chain reaction targeting the mitochondrial cytochrome $\mathrm{b}(c y t-b)$ gene of Haemoproteus and Plasmodium [18] with $3 \mathrm{ng} / \mu \mathrm{L}$ of sample DNA, $0.6 \mu \mathrm{M}$ of each primer, and GoTaq Green Master Mix 2x (M7122, Promega). Blood samples from chicken experimentally infected with Plasmodium gallinaceum and samples from chickens raised in arthropod-free environments were used as positive and negative controls, respectively. Gel electrophoresis was conducted to visualize amplification products, using $2 \%$ agarose gel, SYBR Safe (S33102, Invitrogen), and a high-resolution imaging system (Gel Doc EZ System 170-8270, Bio-Rad). PCR amplification products of positive samples were purified with Polyethylene Glycol 8000. Bi-directional sequencing with dye-terminator fluorescent labeling was performed through automated sequencing (ABI Prism 3100, Applied Biosystems); forward and reversed chromatograms were edited and consensus sequences were deposited in GenBank (Additional file 1).

Phylogenetic relationships among Plasmodium lineages identified in this study and related hemosporidian parasites were inferred by using sequences from reference lineages from the MalAvi database [19], for which species was identified based on studies using morphological evidence, as well as penguin-infecting Plasmodium lineages from published studies (Additional file 1). Sequences were aligned using ClustalW [20] as implemented in MEGA 5.2.2 [21]. A Bayesian phylogenetic tree for the parasite sequences was produced using MrBayes 3.2.2 [22] with the GTR + I + G model of nucleotide evolution, as recommended by ModelTest [23]. We ran two Markov chains simultaneously for 5 million generations that were sampled every 1000 generations. The first 1250 trees $(25 \%)$ were discarded as a burn-in step and the remaining trees were used to calculate the posterior probabilities.

\section{Statistical analysis}

"Apparent prevalence" was defined as the number of positive individuals divided by the number of individuals tested. "Survival ratio" was defined as the number of "survivor" individuals divided by the total number of individuals in a given data subset. "Lethality" was defined as the number of "deceased" individuals divided by the total number of individuals infected by a given Plasmodium lineage.

Chi-Square test was used to compare diagnosis (dependent variable - DV) among laboratory methods (independent variable - IV) (TBS + PCR, PCR, TBS; histopathology was not included due to small sample size).

All subsequent analyses in this subsection were restricted to data obtained from systematically sampled and PCR-tested individuals. True prevalence was estimated from apparent prevalence using Blaker's 95\% confidence interval [24] assuming a $80 \%$ sensitivity $[25,26]$ and $100 \%$ specificity.

Mann-Whitney tests were used to determine if warm season period or intake masses (DV) were different between categories of location and oiling (IV). Linear regression was used to determine if there was correlation between warm season period and intake masses. Fisher's exact test was used to compare survival (DV) between categories of diagnosis (IV), either overall or within data subsets. All tests were two-tailed and used a significance level of 0.05 .

Binary logistic regression was employed to determine which independent variables (IV) such as location, warm season period, oiling and intake mass had a significant effect in determining diagnosis (DV). The order of inclusion of variables in the logistic model and best model selection was based on the $P$-value of the Slope-equalto-zero test (only variables with $P>0.1$ were included) and Pearson's Goodness-of-fit test.

All procedures in this study were approved by the Ethics Committee on Animal Use of the School of Veterinary Medicine and Animal Science of the University of São Paulo (CEUA 601415) and were authorized by Brazilian authorities (SISBIO 20825-6).

\section{Results}

Epidemiology of Plasmodium in penguins at rehabilitation centers

Forty-four of the 774 MPs (5.68\%) undergoing rehabilitation at facilities in six states along the coast of Brazil were identified as positive to Plasmodium (Figure 1 and Table 1). Positive individuals were identified at all states except São Paulo, and no individuals were positive upon intake. In all positive cases, clinical history and diagnostic results were consistent with the hypothesis that infection occurred during the stay at rehabilitation facilities. Details on individual rehabilitation history and clinical parameters of Plasmodium-positive individuals are provided in Additional file 2. A substantial proportion of positive cases (39\%: 17/44) were concentrated in a single outbreak that occurred at Santa Catarina in March-April 2009. All avian malaria cases were first identified as positive between the months October and April, inclusive (Figure 2). 


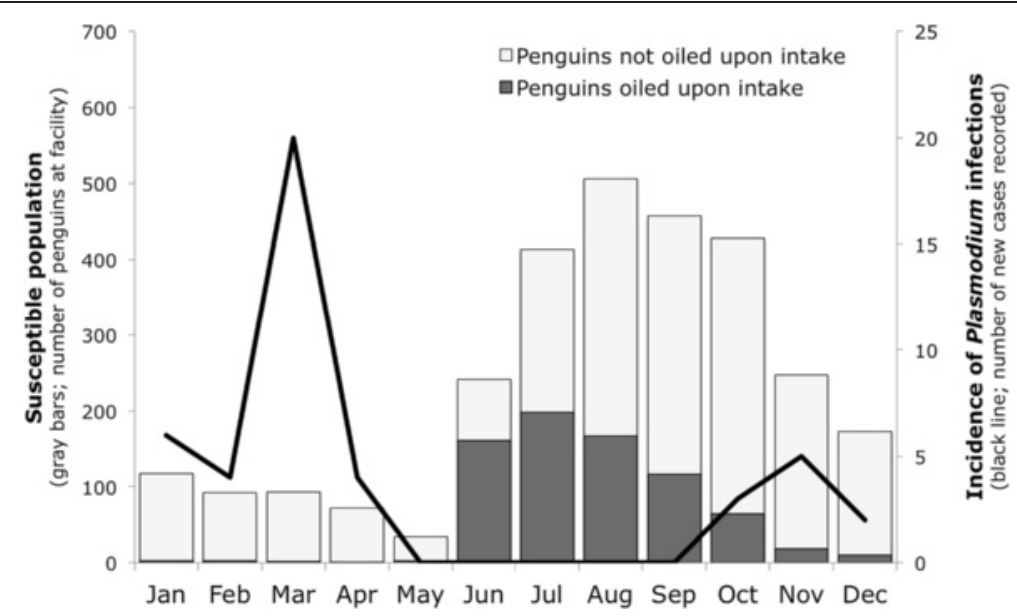

Figure 2 Monthly distribution of Plasmodium infections in Magellanic penguins at rehabilitation facilities in Brazil. Line represents the incidence of Plasmodium infections (number of cases first recorded at each month; right vertical axis). Bars represent the susceptible population (number of penguins that spent one or more days at the rehabilitation facilities in a given month; left vertical axis). Data is combined for all facilities and years.

Individuals tested with PCR or with a combination of PCR and TBS were more frequently determined to be positive $(7.50 \%$ and $6.32 \%$, respectively) than those tested with TBS alone $(1.79 \%)(P=0.022)$. All samples identified as positive by TBS were positive in the corresponding PCR test, whereas only $45.5 \%$ (10/22) PCRpositive samples were identified as positive in the corresponding TBS.

When only individuals sampled systematically and tested with PCR (whether in combination with TBS or not) are considered, apparent prevalence was $7.75 \%$ $(30 / 387)$ (Table 2). Based on this result, true prevalence was estimated between $6.6 \%$ and $13.5 \%$. Logistic regression of this data subset $($ Log-likelihood $=-43.029$, $P<0.001$; Pearson Goodness-of-fit test: $X^{2}=145.68$, $\mathrm{df}=321, P>0.99)$ revealed the following variables were significant determinants of positivity to Plasmodium: location (Santa Catarina in relation to Rio Grande do Sul: $\mathrm{Z}=1.75, P=0.080, \mathrm{~b}=1.437, \mathrm{~b}_{\mathrm{C} 195 \%}=-0.172-3.048$, $\left.\mathrm{OR}=4.21, \mathrm{OR}_{\mathrm{CI} 195 \%}=0.84-21.06\right)$, warm season period $\left(\mathrm{Z}=4.97, P<0.001, \mathrm{~b}=0.030, \mathrm{~b}_{\mathrm{C} 195 \%}=0.018-0.042\right.$, $\left.\mathrm{OR}=1.03, \mathrm{OR}_{\mathrm{CI} 195 \%}=1.02-1.04\right)$ and intake mass $(\mathrm{Z}=2.47$, $P=0.013, \mathrm{~b}=0.0014, \mathrm{~b}_{\mathrm{C} 195 \%}=0.0003-0.0025, \mathrm{OR}=1.01$, $\left.\mathrm{OR}_{\mathrm{C} 195 \%}=1.01-1.01\right)$. Oiling did not have a significant effect (not oiled in relation to oiled; $\mathrm{Z}=0.59, P=0.554$, $\mathrm{b}=0.47, \mathrm{~b}_{\mathrm{C} 195 \%}=-1.10-2.05, \mathrm{OR}=1.61, \mathrm{OR}_{\mathrm{CI} 95 \%}=$ $0.33-7.75)$.

Strong association/correlation was present amongst variables: between location and oiling $(P<0.001)$, warm season period $(P<0.001)$, and intake mass $(P=0.029)$; between oiling and warm season period $(P<0.001)$ and intake mass $(P=0.005)$; and between warm season period and intake mass $\left(P=0.001, \mathrm{R}^{2}=0.039, \mathrm{~b}>0\right)$. Survival was significantly different between Plasmodium-positive and
Plasmodium-negative individuals, with $66.6 \%$ of the Plasmodium-positive MPs dying during rehabilitation whereas just $46.21 \%$ of the Plasmodium-negative died $(P=0.037)$.

\section{Plasmodium lineages infecting penguins}

Morphological characterization of parasites revealed the occurrence of at least four different morphospecies in 16 blood smear-positive individuals: $P$. (Novyella) nucleophilum, P. (Haemamoeba) cathemerium, P. (Haemamoeba) tejerai and P. (Huffia) elongatum (photomicrographs provided in Additional file 3 and in references [8] and [14]). In the blood smears of $P$. cathemerium, it should be noted that in addition to the well-defined elongated rod-shaped pigment granules with pointed ends that are unique to this species, unusual morphological characteristics were also noted: meronts were relatively small with scanty cytoplasm and young macrogametocytes frequently presented relatively large vacuoles surrounded by small round pigment granules.

$C y t-b$ sequences were obtained from 34 of the 36 PCRpositive individuals (see Additional file 1), and phylogenetic analysis revealed that these lineages can be classified in 10 distinct clusters (Figure 3). Four clusters were confirmed as morphospecies based on parasite morphology in blood smears: $P$. cathemerium (cluster A), P. nucleophilum (cluster G), P. tejerai (cluster H), and P. elongatum (cluster I). Additionally, even though the morphology of Plasmodium lineage D (obtained from penguin CRAM2125) could not be observed in blood smears, the sequences from this lineage neatly clustered with a reference $P$. (Novyella) unalis lineage, with high probability (100) and sequence identity $(444 / 445$ nucleotides $=99.76 \%)$. Five phylogenetic lineages ( $\mathrm{B}, \mathrm{C}, \mathrm{E}, \mathrm{F}$ and $\mathrm{J}$ ) did not cluster with 
Table 2 Details of the diagnostic results in relation to sample collection and testing strategy, age group, oiling and survival

\begin{tabular}{|c|c|c|c|c|c|c|c|}
\hline \multirow[t]{2}{*}{ Sampling and screening } & \multirow{2}{*}{$\begin{array}{l}\text { Age } \\
\text { group }\end{array}$} & \multirow[t]{2}{*}{ Oiling } & \multicolumn{2}{|l|}{ Died } & \multicolumn{2}{|l|}{ Survived } & \multirow[t]{2}{*}{ Tota } \\
\hline & & & Positive & Negative & Positive & Negative & \\
\hline Systematically sampled & Juvenile & Oiled & 3 & 92 & 0 & 81 & 176 \\
\hline \multirow[t]{3}{*}{ and PCR-tested individuals } & & Not oiled & 13 & 62 & 9 & 67 & 151 \\
\hline & Adult & Oiled & 0 & 5 & 0 & 37 & 42 \\
\hline & & Not oiled & 4 & 6 & 1 & 7 & 18 \\
\hline Opportunistically sampled & Juvenile & Oiled & 1 & 2 & 0 & 9 & 12 \\
\hline \multirow[t]{3}{*}{ and/or non-PCR-tested individuals } & & Not oiled & 7 & 129 & 5 & 213 & 354 \\
\hline & Adult & Oiled & 1 & 5 & 0 & 11 & 17 \\
\hline & & Not oiled & 0 & 0 & 0 & 4 & 4 \\
\hline Total & & & 29 & 301 & 15 & 429 & 774 \\
\hline
\end{tabular}

any reference lineages nor with lineages previously obtained from penguins.

\section{Pathology of avian malaria in penguins}

Twenty-two Plasmodium-positive cases were examined by histopathology, and exoerythrocytic meronts were observed in 19 cases (86.4\%). Meronts were present in macrophages and endothelial cells (Figure 4A), and occurred in a broad variety of tissues, especially in the heart, liver, lungs, spleen and kidneys.

P. tejerai was lethal to $75 \%$ of penguins infected (12/16), and tissue meronts were observed in all six $P$. tejera $i$ cases examined by histopathology. P. cathemerium was identified in 9 MPs, three of which died (33\% lethality); another two were euthanized for other reasons. Only one of three P. nucleophilum-infected MPs died, and necropsy revealed there were no tissue meronts but high numbers of intraerythrocytic parasites were present within blood vessels (Figure 4B); the concurrence of other significant pathological processes (severe splenic amyloidosis, helminthes within lungs and liver parenchyma and intestinal blood vessels) did not allow for a conclusion as to whether or not avian malaria was the cause of death. $P$. elongatum was identified in one dead MP, which did present tissue meronts. P. unalis was identified in one dead penguin, but no tissue meronts were observed and severe respiratory lesions indicated that aspergillosis was the primary cause of death. Plasmodium sp lineages $\mathrm{E}$ and $\mathrm{J}$ were each identified in one individual, and tissue meronts were present in each case. Plasmodium lineage $\mathrm{C}$ was identified in two euthanized individuals; tissue meronts were present in both cases but concurred with other significant lesions (severe aspergillosis accompanied by necrotizing pancarditis; amyloidosis and helminthes within air sacs).

Only one case of mixed infection resulted in death (P. elongatum + P. tejerai), however histopathology was not conducted and therefore the role of avian malaria towards the cause of death could not be determined. Diffuse interstitial pneumonia occurred in all examined cases $(n=22)$ and was most frequently granulocytic (82\%) (Figure 4C). Multifocal hepatitis occurred in all examined cases $(n=20)$ as was most frequently mononuclear (70\%) (Figure 4D); hepatic necrosis and ductal hyperplasia were present in $20 \%$ and $25 \%$ of cases, respectively. Splenitis occurred in $75 \%$ of cases $(n=20)$ and was most often granulocytic $(67 \%)$; necrotizing splenitis and/or lymphocytolysis was observed in 65\% of cases (Figure 4E), and massive splenic hemorrhages were observed in two cases (10\%). Hematopoiesis (60\%) and hemosiderosis (90\%) were frequently observed in the spleen and/or liver $(n=20)$. Myocarditis was observed in $33.3 \%$ of cases $(n=21)$ and was most frequently granulocytic (57\%).

\section{Discussion}

Avian malaria has been widely recognised as one of the most significant infectious diseases for wild and captive penguins $[3,4,27]$. Our findings demonstrate that Plasmodium spp. infect Magellanic penguins at several rehabilitation centers along the Brazilian coast, leading to substantial levels of mortality, and limiting the success of rehabilitation efforts for this species at these centers.

\section{Epidemiology of avian malaria in penguins at rehabilitation centers}

While the overall apparent prevalence ranged from $2.1 \%$ to $13.8 \%$ among rehabilitation centers, when only samples collected and tested in a systematic manner with highly sensitive diagnostic methods were considered, the apparent prevalence was $7.8 \%$. With $95 \%$ confidence, true prevalence was estimated between $6.6 \%$ and $13.5 \%$. The only comparable data in the literature are provided by the Southern African Foundation for the Conservation of Coastal Birds (SANCCOB), an organization that rehabilitates 


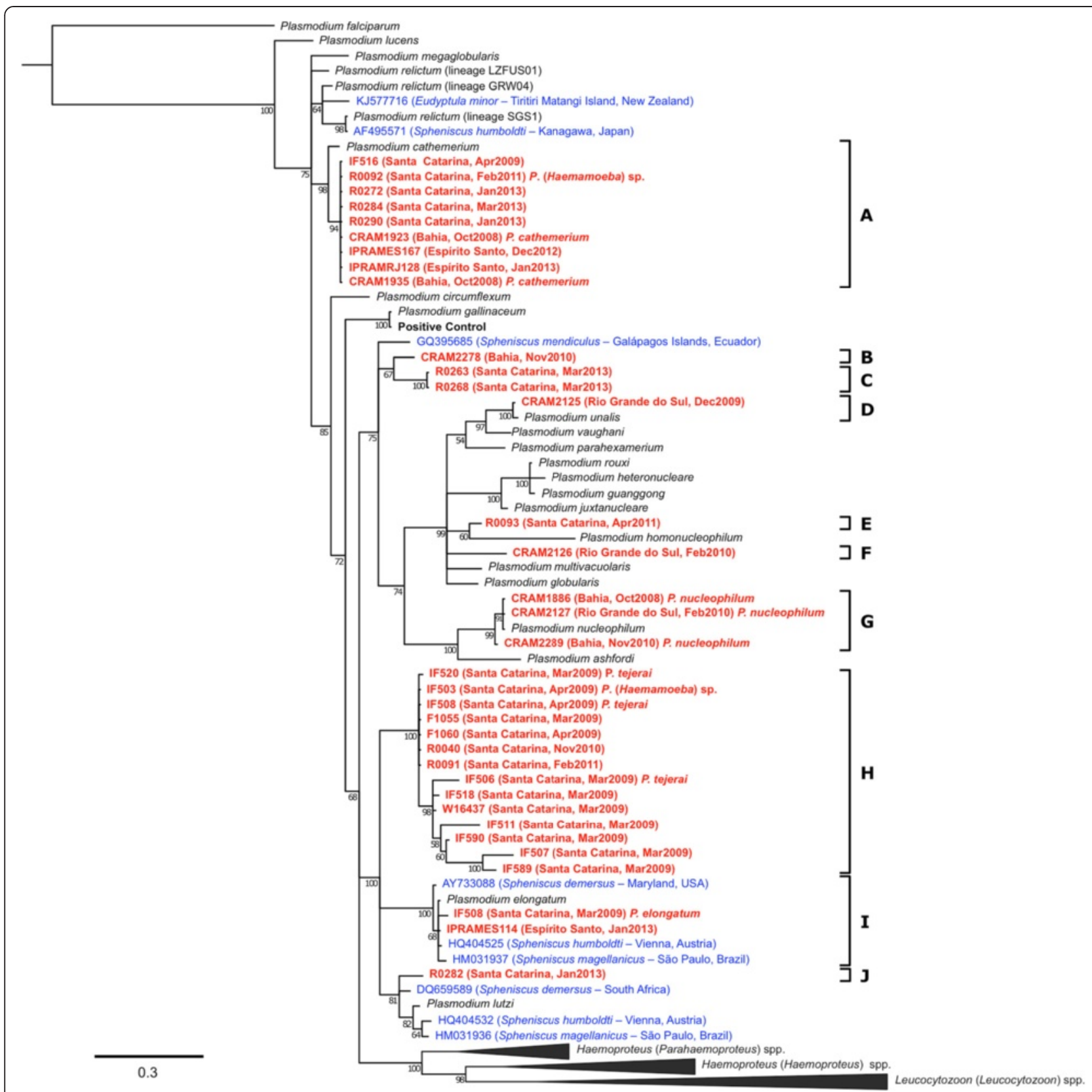

Figure 3 Phylogenetic tree of the Plasmodium spp. lineages identified in penguins. (red) Magellanic penguins undergoing rehabilitation along the coast of Brazil (this study), (blue) published penguin-infecting lineages, (black) reference lineages. Branch lengths are drawn proportionally to the extent of changes (scale bar is shown).

African penguins (Spheniscus demersus) in South Africa; 17$34 \%$ of the African penguins admitted by SANCCOB in 2001-2002 were blood smear-positive to Plasmodium at some point during their rehabilitation [27].

We found that $5 \%$ of MPs that survived through rehabilitation were Plasmodium-positive at some point, compared to $10.8 \%$ of the deceased MPs, with a $44 \%$ higher mortality in Plasmodium-positive penguins compared to Plasmodium-negative (66.6\% vs. $46.2 \%)$. These results contrast with the observed epidemiology at SANCCOB, where the proportion of animals released of Plasmodium-positive individuals is not significantly different from that of the overall rehabilitation population [27].

There is, however, a critical difference to be considered when comparing our findings with those of SANCCOB: there are no records of Plasmodium infections in wild MPs, whereas African penguins are infected in the wild [5]. Therefore, whilst we found no evidence to indicate that the MPs we studied carried the infection from the wild, at SANCCOB $30-35 \%$ of the Plasmodium-positive 


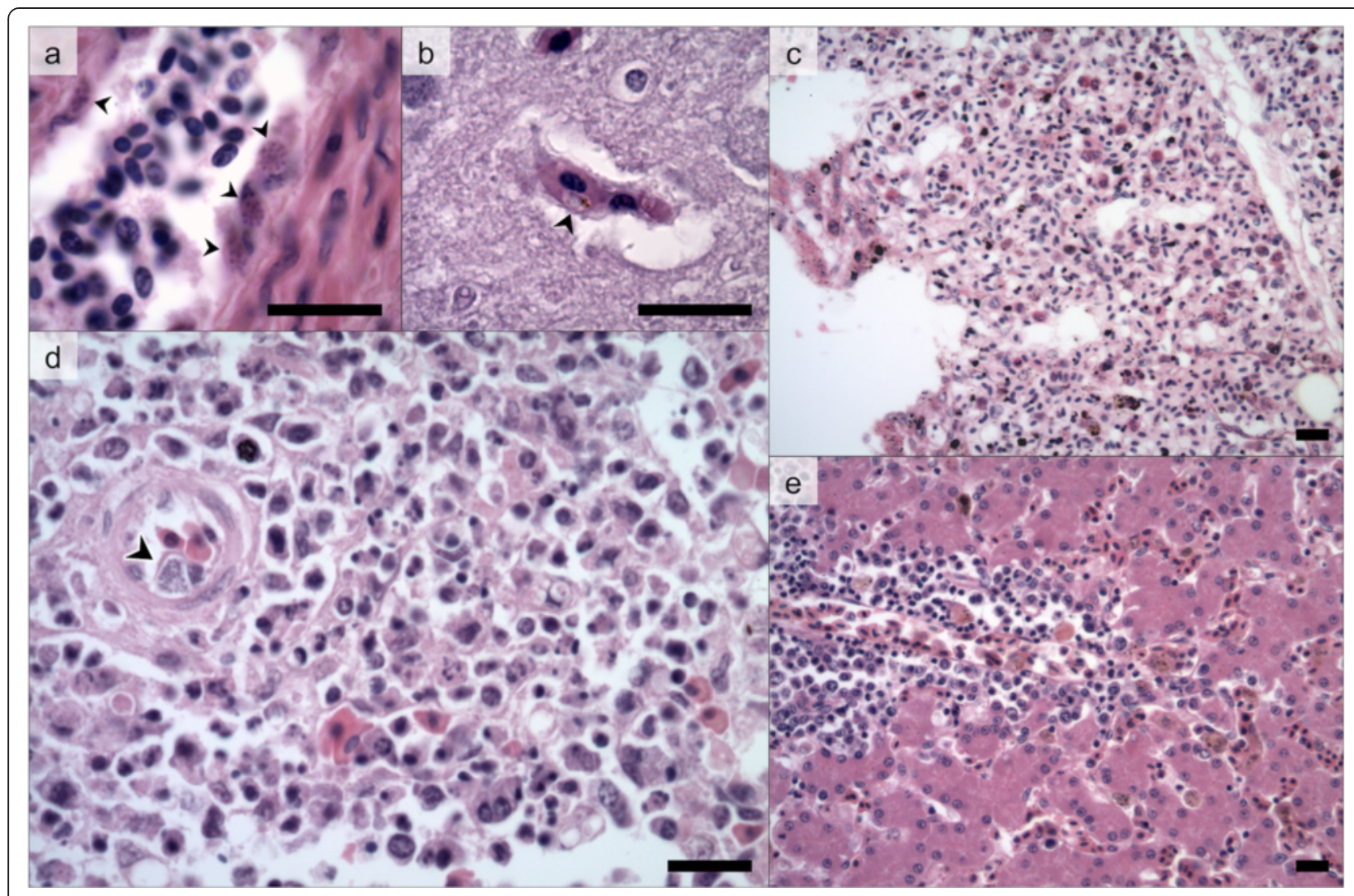

Figure 4 Histological findings associated with avian malaria in Magellanic penguins. (a) exoerythrocytic meronts in endothelial cells (arrowheads) within a liver arteriole (R0040, P. tejerai); (b) parasitized erythrocyte (arrowhead) within a cerebral blood vessel (CRAM2127, P. nucleophilum); (c) diffuse granulocytic interstitial pneumonia, congestion and edema (IF584, P. tejerai); (d) diffuse necrotizing splenitis with an exoerythrocytic meront within an endothelial cell of a central arteriole (arrowhead) (R0290, P. cathemerium); (e) multifocal perivascular mononuclear hepatitis, congestion and hemosiderosis (R0093, Plasmodium sp lineage E). Hematoxilin-Eosin. Scale bars $=15 \mu \mathrm{m}$.

African penguins are already positive upon intake [27]. In this context, the epidemiology of avian malaria in Magellanic penguins in rehabilitation centers may resemble more that of captive penguins in the Northern Hemisphere than that of their African counterparts.

In the Northern Hemisphere, it is well established that the occurrence of avian malaria in penguins is strongly modulated by seasonality, with cases concentrated in the Boreal spring-summer due to climate-mediated fluctuations in mosquito abundance $[10,28,29]$. We observed a consistent concentration of all Plasmodium-positive cases in the Austral spring-summer (October to April), with the probability of infection being positively correlated with the total number of days spent in rehabilitation during that time of the year. In contrast, most MPs are received for rehabilitation along the Brazilian coast during winter months (June to September) (Figure 2), which relates to the species' wintering ecology [9]. Current rehabilitation protocols require the release of MPs is to be avoided during the summer months in recognition of the species' migration [15]. As a result, individuals that were unable to meet the release criteria until spring are retained for release in the subsequent winter; a lower number of individuals are also admitted for rehabilitation during summer.

Surprisingly, body mass upon intake was found to be a positively-correlated predictor of Plasmodium infection, indicating that MPs admitted with higher body mass have a higher probability of becoming infected with Plasmodium. A possible explanation is that MPs admitted during summer months are often admitted due to moult problems and as a result may have relatively higher body mass (RETV, personal observations).

The rehabilitation facility studied in Santa Catarina had a substantially higher Plasmodium incidence than the other facilities involved in this study. The facility is located within a State Park (Parque Estadual do Rio Vermelho) with penguin enclosures directly under tree cover of Atlantic forest and less than 10 metres away from a large freshwater lake (Lagoa da Conceição). This provides an ideal environment for mosquito proliferation and close proximity to an abundant and diverse avifauna. Additionally, this facility rehabilitates not only marine animals but also terrestrial wildlife, including 
birds apprehended from illegal trade. The higher apparent prevalence of MPs at this facility may reflect not a regional difference in susceptibility or lineage virulence, but is more likely to be the consequence of an increased frequency of inoculation due to close contact with mosquitoes and other birds acting as proximal reservoirs of infection.

\section{Plasmodium lineages infecting penguins and their pathology}

It has been generally accepted that $P$. relictum and $P$. elongatum are the two most significant species of Plasmodium responsible for avian malaria in penguins $[3,5,6]$, in addition to an isolated record of P. juxtanucleare [7]. In recent studies $[8,14]$ we have documented the role of $P$. tejerai in causing an avian malaria outbreak in MPs at Santa Catarina.

Our results demonstrate that a broad variety of Plasmodium species can be found in penguins undergoing rehabilitation in South America, including three species that had not yet been demonstrated to infect penguins (P. cathemerium, P. nucleophilum and P. unalis) and 5 lineages that albeit unidentified clearly correspond to Plasmodium species that also have not yet been reported in penguins (lineages B, C, E, F and J). Such diversity of plasmodia corroborates the interpretation that the infection of captive penguins results from local mosquitoes inoculating penguins with Plasmodium spp. acquired from other birds surrounding of the penguin exhibits [12,29-31].

It is worth noting that $P$. cathemerium, $P$. elongatum, $P$. nucleophilum and P. relictum are renowned as generalist parasites with low host-specificity, infecting a broad range of avian species in several taxonomic orders [32]. In this sense, our findings suggest that the predominance of $P$. relictum in Europe and Asia and P. relictum and $P$. elongatum in North America might not necessarily indicate a particular susceptibility of penguins to those parasites, but perhaps reflects their natural abundance in those regions.

Although we observed mature $P$. cathemerium microgametocytes with well-defined elongated rod-shaped pigment granules with pointed ends, which are the defining features of P. (Haemamoeba) cathemerium [16], in all cases we also observed late trophozoites and young macrogametocytes with relatively large vacuoles surrounded by small round pigment granules and relatively small meronts with scanty cytoplasm (Additional file 3), which are uncharacteristic to P. cathemerium. These findings may be interpreted as: (a) a host-specific morphological variation of $P$. cathemerium, (b) a variant or subspecies of $P$. cathemerium, (c) coinfection with a secondary unidentified lineage, or (d) a novel and yet undescribed Plasmodium species whose morphological characteristics overlap with those of $P$. cathemerium. Considering the molecular evidence indicating a consistently high sequence identity and phylogenetic proximity with a reference lineage of $P$. cathemerium, along with a failure to retrieve sequences of another Plasmodium lineage in the same samples, we believe there is sufficient evidence to identify these lineages as $P$. cathemerium and that hypothesis (a) or (b) are most likely.

\section{Pathogenicity of Plasmodium to penguins}

Even though penguins seem to be susceptible to infection by a variety of Plasmodium lineages occurring regionally, we cannot say that all lineages have similar epidemiology or pathogenicity. In this study we found that $P$. tejerai and P. cathemerium were lethal (75\% and 33\% of MPs in which they were detected, respectively) whilst other lineages had only a few cases recorded and/or could not be demonstrated as the leading factor causing death. These findings, combined with previous reports that albeit less frequent $P$. relictum tends to produce more severe disease than P. elongatum in penguins [29,31], raises the question on whether the Plasmodium subgenus Haemamoeba is more pathogenic to these birds than other subgenera of Plasmodium. Comparative pathology through experimental inoculation in domestic birds may assist in clarifying whether pathogenicity is intrinsically higher for these lineages or if it reflects a susceptibility bias present in penguins.

Overall, however, the histopathological lesions observed in this study were generally consistent among lineages. The most prominent pathological processes were granulocytic pneumonia and splenitis and mononuclear hepatitis; these were probably the effect of vasculitis associated with the proliferation of Plasmodium within endothelial cells of these tissues. In most cases, death likely culminated as a result of respiratory insufficiency from the marked pneumonia, congestion and edema. These lesions are not unlike those reported in $P$. relictum and $P$. elongatum infections in penguins in zoos and aquaria in the Northern Hemisphere [10,11,33].

In contrast, no exoerythrocytic meronts were observed in the tissues of the only MP that died with a P. nucleophilum infection, whereas a high number of intraerythrocytic parasites was observed within blood vessels. Similarly, the only individual infected with $P$. unalis did not present detectable exoerythrocytic meronts. This distinct pattern may indicate a different stage of infection or a distinct pathogenesis. Future studies will be welcome to clarify whether this is a consistent pattern in infections by these lineages in penguins and which pathophysiological mechanisms are involved.

\section{Concurrent diseases}

Two of the Plasmodium-positive MPs identified in this study had also been identified as positive to Avipoxvirus in a previous study [34]. This is probably not uncommon as both pathogens are mosquito-borne, however it may 
confuse interpretation of pathological findings. In particular, the only two individuals studied by Niemeyer et al. [34] that presented necrotizing splenitis were found to be Plasmodium-positive in this study. This might indicate that such severe lesions were related to avian malaria and not poxvirosis, and that Avipoxvirus might not have been as pathogenic as originally thought.

Other concurrent diseases included aspergillosis, gastrointestinal helminthiasis, spleen amyloidosis, cholestasis, unidentified myocardium cysts, and helminthes in the lungs, liver, air sacs and skin (Additional file 3). Some of these findings, such as aspergillosis and gastrointestinal helminthiasis have been previously reported in penguins with avian malaria $[10,35]$. However, there have been no reports of helminthes in the respiratory system, skin or air sacs of MPs [3]. Furthermore, the myocardium cysts herein observed clearly were not Plasmodium and could correspond to either protozoan or metazoan parasites. Additional studies will be conducted to clarify the identity and significance of these parasites.

\section{Implications for rehabilitation and conservation}

The prevention of avian malaria in penguins in Northern Hemisphere zoos has largely relied on the oral administration of primaquine during summer [15]. In Brazil, primaquine commerce is restricted by the government due to concerns of potential resistance in human malaria, and therefore this drug cannot be acquired or used by rehabilitation centers. As a result, the centers are forced to employ other prevention strategies, namely the physical isolation of penguins from mosquitoes, which is often challenging and costly.

Our study sheds light on a positive aspect of the epidemiology of this disease at rehabilitation centers, namely that the periods in which Plasmodium infections occur (summer) is directly opposite to the period in which there are the most penguins in rehabilitation (winter). Consequently, one of the key strategies for the prevention of avian malaria in these facilities might be to develop rehabilitation protocols that allow the shortening of the time needed by the penguins to achieve the fit-to-release criteria, so that they can be released before the summer. As a result, these facilities would benefit from narrowing their malariaprevention efforts to a relatively lower number of individuals (those received and/or maintained between October and April), becoming more effective in the prevention and early diagnosis. This is a relevant implication not only for permanent rehabilitation efforts, but also for oil spill responses involving penguins, when the physical and human resources required for malaria prevention, diagnosis and treatment may be substantial [35] and potentially beyond the capacity of those involved.

A number of MPs herein examined were released despite having been Plasmodium-positive at some point.
These individuals were clinically healthy and passed the standard release criteria [15] and were blood smearnegative or, in a few cases, blood samples were collected but not examined in time before release. In the cases where blood smears were negative, it must be considered that even non-parasitemic penguins can relapse if treated with corticosteroids - and presumably the same would occur if they became stressed - due to the persistence of exoerythrocytic meronts [30]. In the cases where samples could not be tested before release, this exposes a potential dillema that is common in oil spill responses, where it is not always feasible to test the large numbers of individuals in a brief period [36,37].

Brossy et al. [38] expressed concern on the potential of rehabilitation centers releasing African penguins with blood parasites, and perhaps this concern should be even greater for MPs considering that Plasmodium has yet to be recorded in this species in the wild. In the case of MPs, however, because climate and environmental conditions are generally adverse and mosquitoes occur very scarcely in the southeastern coast of Argentina [39,40] and are absent at the Malvinas-Falkland Islands [41], the probability of Plasmodium transmission from a rehabilitated penguin to a wild penguin in these regions is very low. Even so, it is important to emphasize that pathogen spillover to wild populations should remain a prime and critical concern for rehabilitation centers, and that even a low non-zero probability is nonetheless a significant risk to be considered and addressed.

Rehabilitation facilities on the Pacific coast of South America may be in a different situation. There are reports of avian malaria in MPs undergoing rehabilitation in Chile [13], and even though no studies have detected blood parasites in penguins in Chile [5,6], both ecological models [40] and blood parasite studies in other avian species [42] consistently indicate that mosquitoes are abundant on the Southwestern coast of South America. Studies examining the occurrence of blood parasites in the Chilean populations of MPs are therefore urgently required, and rehabilitation facilities in the region should remain cautious of potentially releasing Plasmodium-positive individuals back into the wild.

\section{Additional files}

Additional file 1: Public database ascension numbers. Genbank and MalAvi ascension numbers for the sequences obtained or included in the analyses. Taxonomic names within brackets indicate the taxon to which the species is presumed to correspond on the basis of phylogenetic analyses of the cytochrome $b$ mitochondrial gene.

Additional file 2: Individual details of Plasmodium-positive Magellanic penguins. Spreadsheet with rehabilitation history and clinical parameters of Plasmodium-positive Magellanic penguins identified in this study.

Additional file 3: Plasmodium spp. in Giemsa-stained blood smears of Magellanic penguins. Photomicrographs: P. nucleophilum (CRAM2127): 
$(\mathrm{a}, \mathrm{b})$ trophozoites, $(\mathrm{c}, \mathrm{d})$ meronts, (e) coinfection by erythrocytic meront and microgametocyte, (f) macrogametocyte, (g) microgametocyte, (h) co-infection by macro and microgametocyte; P. cathemerium (CRAM1923): (i) trophozoite, (j,k) meronts, (I-n) macrogametocytes, $(0, p)$ microgametocytes. Scale bar $=5 \mu \mathrm{m}$.

\section{Abbreviations}

DV: Dependent variable; HP: Histopathology; IV: Independent variable; MPs: Magellanic penguins; PCR: nested polymerase chain reaction; SANCCOB: Southern African Foundation for the Conservation of Coastal Birds; TBS: Thin blood smears.

\section{Competing interests}

The authors declare that they have no competing interests.

\section{Authors' contributions}

RETV collected and prepared samples, performed hematological exams, necropsies, molecular testing, histopathological analyses, and drafted the manuscript. RPSF, CKMK and RCCB collected and prepared samples, performed hematological exams and necropsies, and helped to draft the manuscript. VR and MA assisted the epidemiological and statistical analyses and helped to draft the manuscript. SE, FCFJ and EMB assisted the molecular testing, gene sequencing, morphological and phylogenetic analyses, and helped to draft the manuscript. JLCD provided general supervision, participated in the study design and coordination and helped to draft the manuscript. All authors read and approved the final manuscript.

\section{Acknowledgments}

We wish to thank our friends and colleagues at Laboratório de Patologia Comparada de Animais Selvagens, Centro de Recuperação de Animais Marinhos da Universidade Federal do Rio Grande, Associação R3 Animal, Centro de Triagem de Animais Silvestres de Florianópolis, Instituto de Pesquisa e Reabilitação de Animais Marinhos, Fundação Museu de História Pesquisa e Arqueologia do Mar, Centro de Triagem de Animais Selvagens Lello-Unimonte, Instituto de Ciências Biomédicas da Universidade de São Paulo, and Instituto de Ciências Biológicas da Universidade Federal de Minas Gerais for their partnership and contributions to this study. We are grateful to Ângela Cabana, Eric Woehler, Fernanda Colabuono, Melissa Xavier, Monique van Rensburg, Nola Parsons, Renata Hurtado, Ricardo Dias and Ricardo Pereira for their valuable contributions. To Margareth Capurro-Guimarães for providing us the control samples. This study was supported by the São Paulo Research Foundation (FAPESP 2009/53956-9, 2010/51801-5), Minas Gerais Research Foundation (FAPEMIG), Brazilian Federal Agency for the Support and Evaluation of Graduate Education (CAPES), National Counsel of Technological and Scientific Development (CNPq) and National Institute of Science and Technology, Genetic and Health Information of the Brazilian Livestock (INCT-IGSPB).

\section{Author details}

${ }^{1}$ Departamento de Patologia, Faculdade de Medicina Veterinária e Zootecnia, Laboratório de Patologia Comparada de Animais Selvagens, Universidade de São Paulo, Avenida Orlando Marques de Paiva 87, São Paulo, SP, 05088-000, Brazil. ${ }^{2}$ Centro de Recuperação de Animais Marinhos, Museu Oceanográfico Professor Eliézer de Carvalho Rios, Universidade Federal do Rio Grande, Rua Capitão Heitor Perdigão 10, Rio Grande, RS 92200-580, Brazil. ${ }^{3}$ Associação R3 Animal. Rodovia João Gualberto Soares, Entrada do Parque Estadual do Rio Vermelho, Barra da Lagoa, Florianópolis, SC 88061-500, Brazil. "Instituto de Pesquisa e Reabilitação de Animais Marinhos. Rodovia BR 262, Instituto Estadual de Meio Ambiente e Recursos Hídricos, Jardim América, Cariacica, ES 29140-130, Brazil. ${ }^{5}$ International Fund for Animal Welfare, 290 Summer Street, Yarmouth Port, MA 02675, USA. 'Departamento de Análises Clínicas e Toxicológicas, Faculdade de Ciências Farmacêuticas, Universidade de São Paulo, Avenida Professor Lineu Prestes 580, Butantã, São Paulo, SP 05508-000, Brazil.

${ }^{7}$ Departamento de Medicina Veterinária Preventiva e Saúde Animal, Laboratório de Epidemiologia e Bioestatística, Faculdade de Medicina Veterinária e Zootecnia, Universidade de São Paulo, Avenida Professor Orlando Marques de Paiva 87, São Paulo, SP 05088-000, Brazil. ${ }^{8}$ Departamento de Parasitologia, Instituto de Ciências Biológicas, Universidade Federal de Minas Gerais. Caixa Postal 486, Avenida Antônio Carlos 6627, Pampulha, Belo Horizonte, MG 31270-901, Brazil
Received: 7 October 2014 Accepted: 4 February 2015

Published online: 13 March 2015

\section{References}

1. Martinsen ES, Perkins SL (2013) The diversity of Plasmodium and other Haemosporidians: The interesection of taxonomy, phylogenetics and genomics. In: Carlton JM, Perkins SL, Deitsch KW (ed) Malaria parasites: comparative genomics, evolution and molecular biology. Caister Academic Press, Norfolk, pp 1-15

2. Levin II, Parker PG (2011) Hemosporidian parasites: impacts on avian hosts. In: Miller E, Fowler M (ed) Fowler's zoo and wild animals medicine. Elsevier Saunders, Missouri, pp 356-363

3. Clarke JR, Kerry KR (1993) Diseases and parasites of penguins. Kor J Polar Res 4:79-96

4. Jones HI, Shellam GR (1999) Blood parasites in penguins, and their potential impact on conservation. Mar Ornithol 27:181-184

5. Jones HI, Shellam GR (1999) The occurrence of blood-inhabiting protozoa in captive and free-living penguins. Polar Biol 21:5-10

6. Quillfeldt P, Martínez J, Hennicke J, Ludynia K, Gladbach A, Masello JF, Riou S, Merino S (2010) Hemosporidian blood parasites in seabirds: a comparative genetic study from Antartic to tropical habitats. Naturwissenschaften 97:809-817

7. Grim KC, Van der Merwe E, Sullivan M, Parsons N, McCutchan TF, Cranfield M (2003) Plasmodium juxtanucleare associated with mortality in black-footed penguins (Spheniscus demersus) admitted to a rehabilitation center. J Zoo Wildl Med 34:250-255

8. Vanstreels RET, Kolesnikovas CKM, Sandri S, Silveira P, Belo NO, Ferreira Junior FC, Epiphanio S, Steindel M, Braga ÉM, Catão-Dias JL (2014) Outbreak of avian malaria associated to multiple species of Plasmodium in Magellanic penguins undergoing rehabilitation in Southern Brazil. PLoS One 9:e94994

9. Williams TD, Boersma PD (1995) Magellanic penguin (Spheniscus magellanicus). In: Williams TD (ed) The penguins: Spheniscidae. Oxford University Press, Oxford, pp 249-258

10. Fix AS, Waterhouse C, Greiner EC, Stoskopf MK (1988) Plasmodium relictum as a cause of avian malaria in wild-caught Magellanic penguins (Spheniscus magellanicus). J Wildl Dis 24:610-619

11. Ko KN, Kang SC, Jung JY, Bae JH, Kim JH (2008) Avian malaria associated with Plasmodium spp. infection in a penguin in Jeju Island. Kor J Vet Res 48:197-201

12. Bueno MG, Lopez RPG, Menezes RMT, Costa-Nascimento MJ, Lima GFMC, Araújo RAS, Guida FJV, Kirchgatter K (2010) Identification of Plasmodium relictum causing mortality in penguins (Spheniscus magellanicus) from São Paulo Zoo, Brazil. Vet Parasitol 173:123-127

13. Carvajal ER, Alvarado PM (2009) Pesquisa de Plasmodium spp. en pingüinos de Magallanes (Spheniscus magellanicus) de la Región de los Ríos: Malaria aviar como nueva patología de interés en la avifauna local. Boletín Veterinario Oficial 10:1-4

14. Silveira P, Belo NO, Lacorte GA, Kolesnikovas CKM, Vanstreels RET, Steindel M, Catão-Dias JL, Valkiūnas G, Braga ÉM (2013) Parasitological and new molecular-phylogenetic characterization of the malaria parasite Plasmodium tejerai in South American penguins. Parasitol Int 62:165-171

15. Silva-Filho RP, Ruoppolo V (2007) Sphenisciformes. In: Cubas ZS, Silva JCR, Catão-Dias JL (ed) Tratado de animais selvagens - medicina veterinária. Roca, São Paulo, pp 309-323

16. Valkiūnas G (2004) Avian malaria parasites and other haemosporidia. CRC Press, Boca Ratón

17. Gering E, Atkinson CT (2004) A rapid method for counting nucleated erythrocytes on stained blood smears by digital image analysis. J Parasitol 90:879-881

18. Hellgren O, Waldenström J, Bensch S (2004) A new PCR assay for simultaneous studies of Leucocytozoon, Plasmodium, and Haemoproteus from avian blood. J Parasitol 90:797-802

19. Bensch S, Hellgren O, Pérez-Tris J (2009) MalAvi: a public database of malaria parasites and related haemosporidians in avian hosts based on mitochondrial cytochrome $b$ lineages. Mol Ecol Resour 9:1353-1358

20. Thompson JD, Gibson TJ, Plewniak F, Jeanmougin F, Higgins DG (1997) The CLUSTAL_X windows interface: flexible strategies for multiple sequence alignment aided by quality analysis tools. Nucleic Acids Res 25:4876-4882

21. Tamura K, Peterson D, Peterson N, Stecher G, Nei M, Kumar S (2011) MEGA5: molecular evolutionary genetics analysis using maximum likelihood, 
evolutionary distance, and maximum parsimony methods. Mol Biol Evol 28:2731-2739

22. Ronquist F, Huelsenbeck JP (2003) MrBayes 3: Bayesian phylogenetic inference under mixed models. Bioinformatics 19:1572-1574

23. Posada D, Crandall KA (1998) MODELTEST: testing the model of DNA substitution. Bioinformatics 14:817-818

24. Reiczigel J, Földi J, Ózsvári L (2010) Exact confidence limits for prevalence of a disease with an imperfect diagnostic test. Epidemiol Infect 138:1674-1678

25. Jarvi SI, Schultz JJ, Atkinson CT (2002) PCR diagnostics underestimate the prevalence of avian malaria (Plasmodium relictum) in experimentally-infected passerines. J Parasitol 88:153-158

26. Valkiūnas $G$, Zehtindjiev $P$, Dimitrov D, Križanauskiene A, lezhova TA, Bensch $S$ (2008) Polymerase chain reaction-based identification of Plasmodium (Huffia) elongatum, with remarks on species identity of Haemosporidian lineages deposited in GenBank. Parasitol Res 102:1185-1193

27. Parsons NJ, Underhill LG (2005) Oiled and injured African penguins Spheniscus demersus and other seabirds admitted for rehabilitation in the Western Cape, South Africa, 2001 and 2002. Afr J Mar Sci 27:289-296

28. Sladen WJL, Gailey-Phipps JJ, Divers BJ (1979) Medical problems and treatment of penguins at the Baltimore Zoo. Int Zoo Yearbook 19:202-209

29. Beier JC, Stoskopf MK (1980) The epidemiology of avian malaria in black-footed penguins (Spheniscus demersus). J Zoo Anim Med 11:99-105

30. Cranfield MR, Graczyk TK, Beall FB, laleggio DM, Shaw ML, Skjoldager ML (1994) Subclinical avian malaria infections in African black-footed penguins (Spheniscus demersus) and induction of parasite recrudescence. J Wildl Dis 30:372-376

31. Graczyk TK, Cranfield MR, McCutchan TF, Bicknese EJ (1994) Characteristics of naturally acquired avian malaria infections in naive juvenile African black-footed penguins (Spheniscus demersus). Parasitol Res 80:634-637

32. Bennett GF, Bishop MA, Peirce MA (1993) Checklist of the avian species of Plasmodium Marchiafava \& Celli, 1885 (Apicomplexa) and their distribution by avian family and Wallacean life zones. Syst Parasitol 26:171-179

33. Fleischman RW, Squire RA, Sladen WJL, Melby EC, Jr (1968) Malaria (Plasmodium elongatum) in captive African penguins (Spheniscus demersus). J Am Vet Med Assoc 153:928-935

34. Niemeyer C, Favero CM, Kolesnikovas CKM, Bhering RCC, Brandão P, Catão-Dias JL (2013) Two different avipoxviruses associated with pox disease in Magellanic penguins (Spheniscus magellanicus) along the Brazilian coast. Avian Pathol 42:546-551

35. Griner LA, Sheridan BW (1967) Malaria (Plasmodium relictum) in penguins at the San Diego Zoo. Vet Clin Pathol 1:7-17

36. Wolfaardt AC, Underhill LG, Altwegg R, Visagie J, Williams AJ (2008) Impact of the Treasure oil spill on African penguins Spheniscus demersus at Dassen Island: case study of a rescue operation. Afr J Mar Sci 30:405-419

37. Holcomb J, Callahan B: Management of the Treasure Oiled Wildlife Response Cape Town, South Africa, 2000. In Proc 7th Int Effects Oil Wild Conf: 14-16 October 2003; Hamburg, Germany: International Bird Rescue and Research Center and International Fund for Animal Welfare

38. Brossy JJ, Plös AL, Blackbeard JM, Kline A (1999) Diseases acquired by captive penguins: what happens when they are released into the wild? Mar Ornithol 27:185-186

39. Mitchell CJ, Darsie RF (1985) Mosquitoes of Argentina: Part II. Geographic distribution and bibliography (Diptera, Culicidae). Mosq Syst 17:279-360

40. Walter Reed Biosystematics Unit: VectorMap: Know the vector, know the threat. [http://www.vectormap.org/]

41. Medlock JM, Schaffner F, Fontenille D: Invasive mosquitoes in the European associate continental and overseas territories. [http://www.ecdc.europa.eu/ en/activities/sciadvice/_layouts/forms/Review_DispForm.aspx? $\mathrm{ID}=212 \&$ List=a3216f4c-f040-4f51-9f77-a96046dbfd72]

42. Merino S, Moreno J, Vásquez RA, Martínez J, Sánchez-Monsálvez I, Estades CF, Ippi S, Sabat P, Rozzi R, McGehee S (2008) Haematozoa in forest birds from southern Chile: Latitudinal gradients in prevalence and parasite lineage richness. Austral Ecol

33:329-340

\section{Submit your next manuscript to BioMed Central and take full advantage of:}

- Convenient online submission

- Thorough peer review

- No space constraints or color figure charges

- Immediate publication on acceptance

- Inclusion in PubMed, CAS, Scopus and Google Scholar

- Research which is freely available for redistribution

Submit your manuscript at www.biomedcentral.com/submit 